\title{
Observations of star formation triggered by H II regions
}

\author{
Lise Deharveng $^{1}$ and Annie Zavagno ${ }^{1}$ \\ ${ }^{1}$ Laboratoire d'Astrophysique de Marseille (UMR 6110, CNRS \& Université de Provence) \\ 38 rue F. Joliot-Curie, 13388 Marseille Cedex 13, France \\ email: lise.deharveng@oamp.fr
}

\begin{abstract}
Observations show that expanding $\mathrm{H}$ II regions may trigger star formation. We discuss several aspects of this type of star formation, and try to estimate its prevalence. We show how LMC H II regions may help us to understand what we see in our Galaxy.
\end{abstract}

Keywords. stars: formation, ISM: H II regions, ISM: bubbles, infrared: ISM, infrared: stars

\section{Introduction}

Massive stars are often presented as having a negative feedback on their surroundings in terms of star formation. Via ionization of the gas, and via stellar winds and supernovae explosions at the end of their life, they may disperse their parental molecular cloud, thereby preventing the formation of subsequent stars. Numerous surveys are now available, at all wavelengths, allowing a better understanding of the vicinity of $\mathrm{H}$ II regions, with improved detection of young stellar objects (YSOs). These observations show that massive stars may also provide positive feedback affecting star formation, via the accumulation of neutral material at the periphery of $\mathrm{H}$ II regions, or via the compression of pre-existing dense condensations as they are reached by ionization fronts. In the following, we will give examples of the accumulation of neutral material around expanding $\mathrm{H}$ II regions. We will discuss the various processes able to trigger star formation, how they can be identified, and what kind of stars they form.

\section{The accumulation of neutral material around expanding $H_{\text {II }}$ regions}

This point concerns the accumulation of neutral material around an $\mathrm{H}$ II region, in a layer where the conditions for star formation are fulfilled.

Massive OB2 stars ionize the surrounding material, forming H II regions. Due to the high pressure of the warm ionized gas with respect to that of the cold surrounding neutral material, H II regions expand (Dyson \& Williams 1997). During their expansion, neutral material accumulates between the ionization front (IF) and the shock front (SF) which precedes it on the neutral side. These layers of accumulated material are dense, and with time they may become massive. We can use a very rough model of expansion in a homogeneous medium to get an order of magnitude. For example an O6V (B0V) star evolving in a medium of $10^{3} \mathrm{~cm}^{-3}\left(10^{2} \mathrm{~cm}^{-3}\right)$ will form, after one megayear, an $\mathrm{HII}$ region of radius $5 \mathrm{pc}$ (same size) and of mass $900 M_{\odot}\left(90 M_{\odot}\right)$, surrounded by a neutral shell of $\sim 17000 M_{\odot}\left(\sim 1700 M_{\odot}\right)$. 
How can we observe these shells of collected material?

- We can observe the CO emission of the shells. Knowledge of the velocity allows us to consider only the molecular material associated with the central H II regions. But the $\mathrm{CO}$ emission is not sensitive to high densities; it may be optically thick and, also, in cold dense condensations the CO molecules condense onto the dust grains so that CO may be heavily depleted (Caselli et al. 1999; Bacmann et al. 2002). All these effects result in underestimation of the masses of the shells.

An illustration is given by the Sh $104 \mathrm{H}$ II region (Deharveng et al. 2003); excited by an 06 star, it has a radius $\sim 4 \mathrm{pc}$ and a mass $\sim 450 M_{\odot}$. It is completely surrounded by a shell of molecular material with a mass of $\sim 6000 M_{\odot}$. This shell contains four dense fragments (mapped in CS) elongated along the IF. A deeply embedded and probably young cluster, exciting a compact H II region, is observed in the direction of the most massive fragment.

Many other examples can be found in Beaumont \& Williams (2010). They mapped the $\mathrm{CO}(3-2)$ emission in the direction of 43 bubbles from Churchwell et al.'s catalogue (2006; hereafter CHU06). These bubbles detected at $8 \mu \mathrm{m}$ enclose classical H II regions. Most of these bubbles are surrounded by shells of CO emitting material.

- We can observe the (sub)millimeter thermal emission of the cold dust associated with dense molecular material. But we do not have the velocity information and thus the association may be uncertain.

This is illustrated by the RCW 79 and RCW 120 H II regions. Zavagno et al. (2006) have shown that RCW 79 is surrounded by a shell of cold dust observed at 1.2-mm; the mass of this shell is estimated to be $>3600 M_{\odot}$. RCW 120 has been observed at $1.2-\mathrm{mm}$ (Zavagno et al. 2007) and at $870 \mu \mathrm{m}$ (Deharveng et al. 2009). It is surrounded by a shell of cold dust, with a mass of $\sim 2000 M_{\odot}$. In both objects massive fragments are elongated along the IF, and contain young stellar objects.

Many other examples can be found in Deharveng et al. (2010; hereafter DEH10) who studied the distribution of the cold dust in the direction of 102 bubbles from CHU06, using the ATLASGAL survey of the Galaxy at $870 \mu \mathrm{m}$ (Schuller et al. 2009). This survey
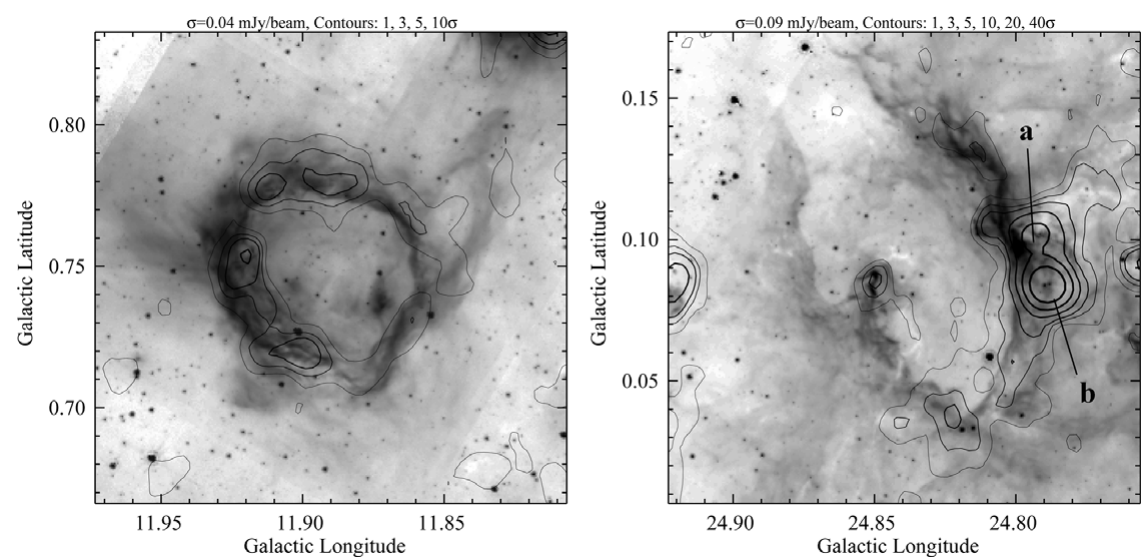

Figure 1. N4 (left) and N36 (right) bubbles from the CHU06 catalogue. The $870 \mu \mathrm{m}$ cold dust emission is presented as contours superimposed on the Spitzer image at $8 \mu \mathrm{m}$; these two bubbles enclose classical H II regions (see DEH10 for details). N4 is surrounded by a shell of collected material, with a mass of $\sim 1150 M_{\odot}$. N36 also shows a faint $870 \mu \mathrm{m}$ emission shell following the bubble, probably formed of collected material, but the massive a and b condensations were probably pre-existing condensations reached by the IF. Condensation a contains a compact H II region and condensation $\mathrm{b}$ contains two $\mathrm{UC} \mathrm{H}$ II regions and various masers. 
covers the inner Galactic plane, $l=300^{\circ}$ to $60^{\circ},|b| \leqslant 1.5$, with an angular resolution of $199^{\prime \prime} 2$ and an rms noise $\sim 0.06 \mathrm{Jy} /$ beam. DEH10 find that at least $86 \%$ of these bubbles, detected at $8 \mu \mathrm{m}$, enclose $\mathrm{H}$ II regions detected thanks to their radio-continuum emission. Considering only the 65 bubbles, all enclosing $\mathrm{H}$ II regions, for which the angular resolution is enough to study the distribution of the associated cold dust, they find that $38 \%$ of these bubbles are surrounded by a shell of collected material; $31 \%$ more bubbles are surrounded by numerous dust condensations, but DEH10 are unable to estimate if they were pre-existing and reached by the ionization fronts or if they formed out of collected material. Fig. 1 shows the N4 and N36 bubbles and their associated cold dust condensations. The association is based on velocity measurements (see details in DEH10).

\section{Are the collected neutral shells expanding?}

It is hard to answer this question. So far there are few observations concerning the expansion of the shells, and they give contradictory results. Examples of expansion can be found in Patel et al. (1995; the IC1396 H II region presents a ring of CO material, of radius $12 \mathrm{pc}$, expanding with a velocity of $5 \mathrm{~km} \mathrm{~s}^{-1}$ ), in Dent et al. (2009; in the Rosette Nebula, a CO ring is also identified, expanding with a velocity $\sim 15 \mathrm{~km} \mathrm{~s}^{-1}$ ), in Kang et al. (2009; a CO shell, expanding with a velocity $\sim 3-4 \mathrm{~km} \mathrm{~s}^{-1}$, surrounds the N102 bubble).

On the other hand, Beaumont \& Williams (2010) do not detect CO emission in the direction of the centers of their bubbles. If the bubbles were surrounded by spherical expanding shells faint blue- and redshifted emissions would be expected in the direction of the central regions. As this is not the case, they conclude that the parental molecular clouds are somewhat flat, and that the bubbles are not three dimensional spherical structures but two dimensional rings.

\section{Different processes for triggering star formation around $\mathbf{H}$ II regions}

Different processes can trigger star formation around H II regions. We will discuss here the two processes most often mentioned. A sketch of the various processes and more details about them can be found in DEH10.

The radiation driven compression of pre-existing condensations.

The H II region expands into a medium where pre-existing dense condensations are present. What happens when one of them is reached by the IF? If the pressure of the ionized gas in the H II region is higher than that inside the molecular condensation an IF progresses inside the condensation, preceded by an SF. A dense ionized boundary layer forms at the periphery of the condensation, and a layer of compressed neutral material accumulates between the two fronts. This layer may fragment and collapse. Alternatively, the whole condensation may implode; thus the name of this process: "radiation-driven implosion of a globule". Star formation follows.

What are the morphological signatures of such a process? First, the condensations are dense. If they are situated in front of an optically visible $\mathrm{H}$ II region they are seen in absorption. Also they are bordered by a bright emission zone ( $\mathrm{H} \alpha$ or radio-continuum emission of the dense ionized gas at their border, or $8 \mu \mathrm{m}$ emission of polycyclic aromatic hydrocarbons (PAHs) in the photodissociation region); thus the name "bright rim clouds" (BRCs). Generally, their velocity differs from that of the IF, and they protrude inside the ionized region. 
A sample of 89 BRCs has been selected on the basis of a high extinction zone bordered by a bright rim at $\mathrm{H} \alpha$, and the presence in this direction of an IRAS source with the colors of an YSO (Sugitani et al. 1991, 1994). Most of these BRCs have been observed at several wavelengths (for example, Thompson et al. 2004, Morgan et al. 2004 for radiocontinuum observations; Sugitani et al. 2000 for millimeter observations; Morgan et al. 2008 for sub-millimeter and IR observations; De Vries et al. 2002, Morgan et al. 2009 for CO observations; Urquhart et al. 2009 for CO and IR observations). These observations allow to compare the pressure in the ionized gas and in the BRC. The conclusion is that about $55 \%$ of the BRCs of the sample are good candidates for triggered star formation (Morgan et al. 2009, Urquhart et al. 2009). Most of the second-generation sources have low or intermediate masses (depending on their luminosity); however a few BRCs harbor a massive second-generation source (see Fig. 3).

Small-scale sequential star formation is a characteristic of star formation in the vicinity of many BRCs. BCR37, on the border of the IC $1396 \mathrm{H}$ II region, is a good illustration of this characteristic. As one gets away from the first-generation star exciting the large $\mathrm{H}$ II region, he finds: i) on the ionized side of the BRC, thus outside the molecular core, some $\mathrm{H} \alpha$-emission stars (Ikeda et al. 2008); these are believed to be $\mathrm{T}$ Tauri stars, thus YSOs of about one megayear; ii) inside the molecular core, two sources with a near-IR excess, one corresponding to an IRAS source. Spitzer-IRAC images show that these two sources are bright at longer wavelengths. One of them is associated with $\mathrm{H}_{2}$ jets and a $\mathrm{CO}$ outflow of dynamic age 0.3 Myr (Duvert et al. 1990), the other is associated with a water maser (Valdettaro et al. 2008). Thus, in the vicinity of this BRC we see a gradient of age among the second-generation sources. Star formation has progressed in time and space from the first generation massive star to the present location of the molecular core, thus confirming its triggering by the expanding $\mathrm{H}$ II region. Also it shows that star formation has not occurred during an implosion (an unique event) but more probably that stars have formed in the compressed layer progressing inside the cloud, as the cloud got ionized.

The collect and collapse process of star formation.

Another mechanism of star formation is the gravitational fragmentation and subsequent collapse of the neutral collected shell surrounding an H II region.

What are the signatures of such a process? First, the fragments are massive and elongated along the IF. Since the collected shell has the same velocity as the IF, the fragments also have the same velocity and do not protrude inside the ionized region. The young sources formed in these fragments share the velocity of the gas from which they form, and they are observed later on in the direction of the collected layer.

The RCW $120 \mathrm{H}$ II region, at the center of an almost perfect bubble (see details in Zavagno et al. 2007 and Deharveng et al. 2009), illustrates such a process. This H II region is surrounded by a shell of collected material of about $2000 M_{\odot}$, which is fragmented; it probably expands in a medium with a density gradient. Two massive fragments, which are elongated along the IF, are present south of RCW 120; they do not protrude inside the ionized region. The more massive one $\left(\sim 1000 M_{\odot}\right.$; Fig. 2$)$ contains several YSOs of various masses and in various evolutionary stages: i) a chain of about 10 class I-class II sources, parallel to the IF, regularly spaced (separation $\sim 0.1 \mathrm{pc}$ ), with an uncertain mass, possibly $\sim 1 M_{\odot}$. The formation of these YSOs probably results from small-scale gravitational instabilities in the collected layer; ii) a massive core, which appears as a resolved source at $100 \mu \mathrm{m}$ (Herschel image, Zavagno et al. 2010a). It contains a class 0 source, of relatively high mass $\left(8-10 M_{\odot}\right)$, with a high accretion rate $\left(\geqslant 10^{-3} M_{\odot} \mathrm{yr}^{-1}\right)$. This massive class 0 YSO is a very good candidate for massive-star formation by collect \& 
collapse. Many other YSOs of low or intermediate mass are observed all around RCW 120, in other condensations, possibly formed by different processes.

\section{What is the prevalence of massive-star formation triggered by $\mathrm{H}_{\mathrm{II}}$ regions?}

In this section we consider only the formation of massive stars.

It is difficult to answer this question because of the complex morphology of most $\mathrm{H}$ II regions. A beginning of an answer can be obtained if we consider very simple $\mathrm{H}$ II regions, as is done by DEH10. Considering only the 65 bubbles, all enclosing $\mathrm{H}$ II regions, for which the angular resolution is enough to study the distribution of the associated cold dust, they find that: i) seven bubbles show associated UC H II regions and nearby 6.7 GHz methanol masers (considered as a signature of massive-star formation at work) in dust condensations adjacent to their IFs; ii) another six show only UC H II regions; iii) another five show only methanol masers in similar condensations. This result suggest that more than a quarter of the bubbles have triggered the formation of massive objects (either via collect and collapse, or via compression of pre-existing clouds). Therefore, star formation triggered by $\mathrm{H}$ II regions may be a significant process, especially for massivestar formation.

The N36 bubble illustrates such a configuration (Fig. 1). The massive condensation adjacent to N36's IF (probably a pre-existing condensation) contains several H II regions and various masers. The N49 bubble is another candidate for triggered massive-star formation. A shell of neutral material, of $\sim 4000 M_{\odot}$, surrounds the central H ir region. A compact $\mathrm{H}$ II region lies in the direction of the shell. And a massive fragment $\left(\sim 2000 M_{\odot}\right)$ contains two YSOs of intermediate mass along with various masers (see DEH10 and Zavagno et al. 2010b for details).

The masses of the second-generation massive-stars

Fig. 3 compares the spectral types of the first- and second-generation massive stars, in regions candidates for triggered star formation. It shows that the second-generation stars

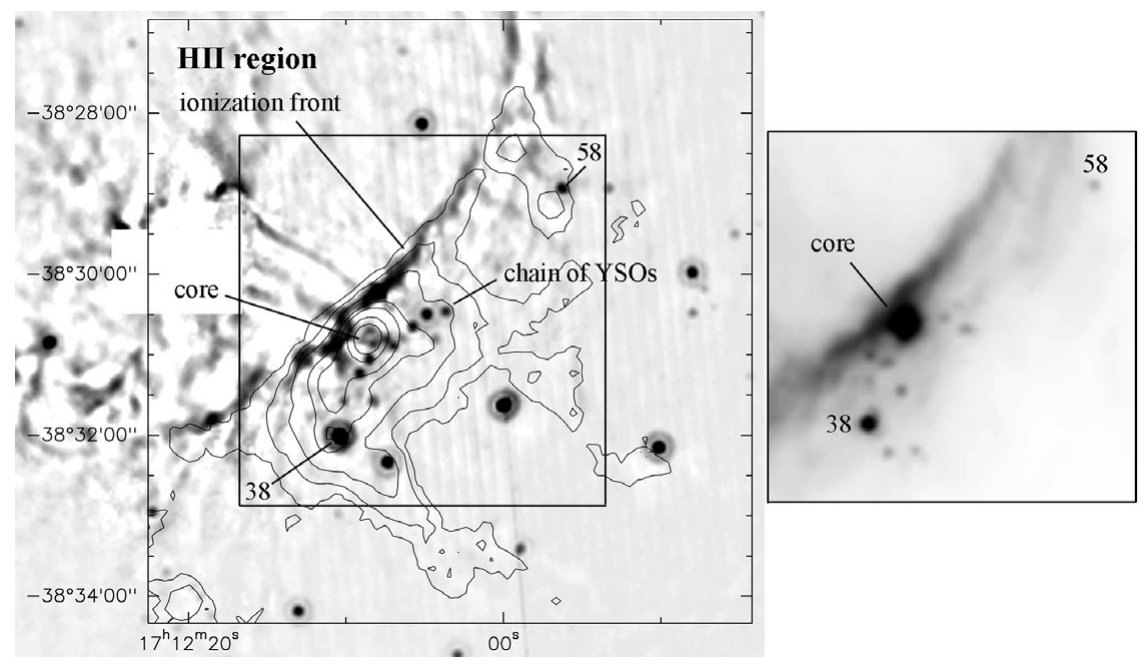

Figure 2. Star formation on the border of RCW 120, in the most massive condensation. Left: the contours show the $870 \mu \mathrm{m}$ emission of the cold dust (APEX-LABOCA observations) superimposed on an unsharp mask image at $24 \mu \mathrm{m}$ (Spitzer-MIPSGAL observations). Right: the same region observed by Herschel-PACS at $100 \mu \mathrm{m}$. 
are less massive than the first-generation ones; this is not a prediction of the collect \& collapse model of star formation, as a late type $\mathrm{O}$ star can accumulate with time a very massive layer of neutral material, which by collapse can form massive fragments and thus massive objects. Also, the second-generation stars are less massive than an O7.5 star.

These points are very important: if it is true that the second-generation stars are always less massive than those of the first-generation, star formation triggered by $\mathrm{H}$ II regions cannot be an important process of massive-star formation. And it cannot explain the formation of early $\mathrm{O}$ stars.

The spectral types of the second generation sources are rather uncertain. They are often estimated from the radio continuum flux of the associated H II regions, assuming a main exciting star, no ionizing photons absorbed by dust, and an optically thin H II region; if it is not the case the spectral types of the second-generation sources are underestimated. Also, it is possible that some of these massive YSOs are still accreting material, and have not yet reached their final masses.

Thus it is very important to identify, if they exist, any good candidate second-generation $\mathrm{H}$ II regions excited by early $\mathrm{O}$ stars. Where can we find them? Possibly at the border of older and larger structures evolving under the influence of stellar winds and supernova explosions, as are present in the Large Magellanic Cloud (see Sect. 6). They are very difficult to detect in our crowded Galactic plane.

\section{Conclusions 1}

Thus, we have seen that:

- The accumulation of neutral material around H II regions is a normal process.

- Stars of all masses form on the borders of H II regions by different processes, either by the compression of pre-existing dense condensations, or by the fragmentation and collapse of the collected layer.

- The formation of massive stars may be triggered by H II regions.

But a few questions are still without a clear answer. Are the bubbles spherical expanding structures, or are they rings formed in flat molecular clouds? Can early O stars be formed by triggering, and in which locations?

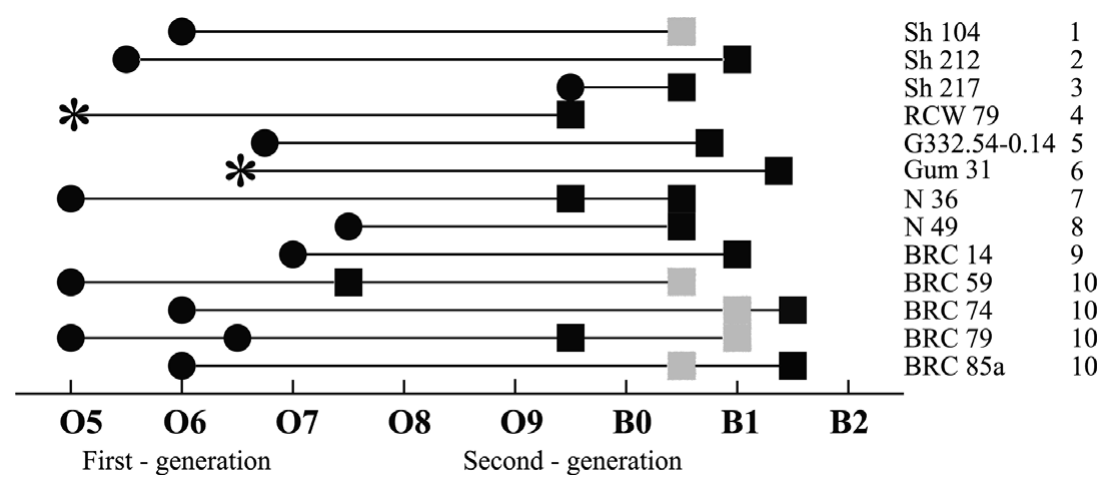

Figure 3. Comparison of the spectral types of the first- and second-generation massive stars. The full circles or asterisks are for the first-generation exciting stars or clusters. The black and grey squares are for the second-generation stars, the spectral types being estimated respectively via the radio flux or via the luminosity of the associated H II regions. References: 1 - Deharveng et al. 2003; 2 - Deharveng et al. 2008; 3 - Brand et al. 2010; 4 - Zavagno et al. 2006; 5 Deharveng, in preparation; 6 - Cappa et al. 2008; 7 - DEH10; 8 - Kantharia et al. 2007; 9 Morgan et al. 2004; 10 - Thompson et al. 2004. 


\section{What can we learn from LMC $\mathrm{H}$ II regions?}

The Large Magellanic Cloud presents us with a unique opportunity to study the interaction of massive stars with their interstellar environment. All the sources are at the same distance. The plane of the LMC is inclined by only $27^{\circ}$ to the plane of the sky, so that the three-dimensional structure of the bubbles can be mapped without confusion. And the reddening is relatively low everywhere. We will consider one example, that of the N44 Hil region $\left(\alpha(2000)=05^{\mathrm{h}} 22^{\mathrm{m}} 25.0^{\mathrm{s}}, \delta(2000)=-67^{\circ} 57^{\prime} 00^{\prime \prime}\right)$.

The brightest part of N44 is a superbubble $(\sim 70 \mathrm{pc} \times 50 \mathrm{pc})$. Many bright H II regions lie at its periphery. This superbubble is expanding with a velocity of $40 \mathrm{~km} \mathrm{~s}^{-1}$. It is a three-dimensional bubble as the two sides of the expanding bubble are observed (Meaburn \& Laspias 1991). The stellar population of this region is well known (OB association LH47/48). The age of the stars inside the superbubble has been estimated to be $\sim 10 \mathrm{Myr}$; the stars situated outside, at the periphery of the superbubble, are younger, with an age of $\sim 5$ Myr (Oey \& Massey 1995). Extended X-ray emission has been detected in this region, from a nearby classical supernova remnant, inside the superbubble, and leaking towards the south-east through a hole in the central bubble. The X-ray emission of the central superbubble is well explained by off-center supernovas exploding in a bubble already formed by the stellar winds of the central cluster (Chu et al. 1993; Magnier et al. 1996). Molecular material, in three massive clouds, is associated with N44 (Yamaghchi et al. 2001; Kim et al. 2004).

Star formation has been studied in this region, based on Spitzer-GLIMPSE and MIPSGAL observations (Whitney et al. 2008; Chen et al. 2009). Sixty YSOs of high or intermediate mass have been identified in the vicinity of N44 (Chen et al. 2009). Eighty percent of the most massive YSOs (types $\mathrm{O}$ and $\mathrm{B}$ ) lie in the direction of $\mathrm{H}$ II regions or in adjacent directions. The highest concentration of such massive YSOs is observed in the direction of the central molecular cloud, where the brightest H II regions are also found. Thus this central region clearly shows three generations of massive stars, with ages of ten, five, and less than one Myr. All the young stellar sources lie in the direction of $\mathrm{H}$ II regions or of their PDRs, suggesting that their formation has been triggered.

A number of YSOs, some characteristic of massive stars, lie in the outskirts of the $\mathrm{H}$ II region and very far from the central superbubble. However, deep $\mathrm{H} \alpha$ images show that the ionized gas emission, mainly in the form of filaments, is very extended. This is confirmed by the emission at $8 \mu \mathrm{m}$, mapped by Spitzer, of the polycyclic aromatic hydrocarbons located in the PDR which covers about the same area. We see here the long-distance influence, in terms of star-formation, of the central ionized region.

\section{Conclusions 2}

A few new directions, in the field of star formation triggered by H II regions, seem interesting to follow:

- Look for the long-distance influence of H II regions in terms of star formation, which may explain the formation of apparently isolated YSOs.

- Search for second-generation early O stars. If there are any, they may be found on the border of large structures, like superbubbles, formed via the combined actions of ionization, stellar winds, and supernovae explosions.

- Use Herschel, and especially the Hi-GAL survey (Molinari et al. 2010), to estimate the extent of star formation triggered by H II regions. 
Acknowledgments: We thank all our collaborators, and especially L. Anderson, J. Brand, J. Caplan, M. Cunningham, P. Jones, S. Kurtz, B. Lefloch, F. Massi, M. Pomarès, D. Russeil, and F. Schuller

\section{References}

Bacmann, A., Lefloch, B., Ceccarelli, C. et al. 2002 A $\& A, 389,6$

Beaumont, C.N., Williams, J.P. 2010, ApJ, 709, 791

Brand, J., Massi, F., Zavagno, A., Deharveng, L. 2010, A\&A, submitted

Cappa, C., Niemela, V.S., Amorin, R., Vasquez, J. 2008, A\&A, 477, 173

Caselli, P., Walmsley, C.M., Tafalla, M. et al. 1999, ApJ, 523,165

Chen, C.-H.R., Chu, Y.-H., Gruendl, R.A. et al. 2009, ApJ, 695, 511

Chu, Y.-H., Mac Low, M.-M., Garcia-Segura, G. et al. 1993, ApJ, 414, 213

Churchwell, E., Povich, M.S., Allen, D. et al. 2006, ApJ, 649, 759 (CHU06)

Deharveng, L., Lefloch, B., Zavagno, A. et al. 2003, A\&A, 408, L25

Deharveng, L., Lefloch, B., Kurtz, S. et al. 2008, A\&SA, 482, 585

Deharveng, L., Zavagno, A., Schuller et al. 2009, A\&A, 496, 177

Deharveng, L., Schuller, F., Anderson, L.D. et al. 2010, A 6 A, accepted, (DEH10)

Dent, W.R.F., Hovey, G.J., Dewdney, P.E. et al. 2009, MNRAS, 395, 1805

De Vries, C.H., Narayanan, G., Snell, R.L. 2002, ApJ, 577, 798

Duvert, G., Cernicharo, J., Bachiller, R., Gomez-Gonzalez, J. 1990 A $\& A$, 233, 190

Dyson, J.E., Williams, D.A. 1997 The physics of the interstellar medium

Ikeda, H., Sugitani, K., Watanabe, M. et al. 2008 AJ, 135, 2323

Kang, M., Bieging, J.H., Kulesa, C.A., Lee, Y. 2009, ApJ, 701, 454

Kantharia, N.G., Goss, W.M., Roshi, D. et al. 2007, JApJ, 28, 41

Kim, S., Walsh, W., Xiao, K. 2004, ApJ, 616, 865

Magnier, A.E., Chu, Y.-H., Points, S.D. et al. 1996, ApJ, 464, 829

Meaburn, J., Laspias, V.N. 1991, A\&A, 245, 635

Molinari, S., Swinyard, B., Bally, J. et al. 2010, A\&A, accepted, arXiv:1001.2106

Morgan, L.K., Thompson, M.A., Urquhart, J.S. et al. A\&BA, 426, 535

Morgan, L.K., Thompson, M.A., Urquhart, J.S., White, G.J. 2008, A\&A, 477, 557

Morgan, L.K., Urquhart, J.S., Thompson, M.A. 2009, MNRAS, 400, 1726

Oey, M.S., Massey, P. 1995, ApJ, 452, 210

Patel, N.A., Goldsmith, P.F., Snell, R.L. et al. 1995, ApJ, 447, 721

Schuller, F., Menten, K.M., Contreras, Y. et al. 2009, A\&A, 504, 415

Sugitani, K., Fukui, Y., Ogura, K. 1991, ApJS, 77, 59

Sugitani, K., Ogura, K. 1994, ApJS, 92, 163

Sugitani, K., Matsuo, H., Nakano, M. et al. AJ,119, 323

Thompson, M.A., Urquhart, J.S., White, G.J. 2004, A\&A, 415, 627

Urquhart, J.S., Morgan, L.K., Thompson, M.A. 2009, A\&A, 497, 789

Valdetarro, R., Migenes, V., Trinidad, M.A. et al. 2008, ApJ, 675, 1352

Whitney, B.A., Sewilo, M., Indebetouw, R. et al. 2008, AJ, 136, 18

Yamaguchi, R., Mizuno, N., Mizuno, A. et al. 2001, PASJ, 53, 985

Zavagno, A., Deharveng, L., Comeron, F. et al. 2006, A\&A, 446, 171

Zavagno, A., Pomarès, M., Deharveng, L. et al. 2007, A\& $A, 472,835$

Zavagno, A., Russeil, D., Motte, F. et al. 2010a, A\&AA, accepted, arXiv:1005,1615

Zavagno, A., Anderson, L.D., Russeil, D. et al. 2010b, A\& $A$, accepted, arXiv:1005,1591 